Supporting Information for Manuscript

\title{
Morphology and Pattern Control of Diphenylalanine Self-Assembly via Evaporative Dewetting
}

\author{
Jiarui Chen ${ }^{1}$, Shuyu Qin ${ }^{1}$, Xinglong Wu ${ }^{1, *}$, and Paul K. Chu ${ }^{2}$ \\ ${ }^{1}$ Key Laboratory of Modern Acoustics, MOE, Institute of Acoustics and Collaborative \\ Innovation Center of Advanced Microstructures, National Laboratory of Solid State \\ Microstructures, Nanjing University, Nanjing 210093, P. R. China \\ ${ }^{2}$ Department of Physics and Materials Science, City University of Hong Kong, Tat \\ Chee Avenue, Kowloon, Hong Kong, China
}


Table S1. Dimensionless numbers and the corresponding definitions.

\begin{tabular}{|c|c|c|}
\hline $\begin{array}{l}\text { Dimensionless } \\
\text { quantities }\end{array}$ & Definitions & Notations \\
\hline Rayleigh Number & $R a=\frac{\rho_{0}^{*} \beta d^{4} \xi_{0}^{*} g}{\kappa_{0}^{*} \mu_{0}^{*}}$ & \multirow{3}{*}{$\begin{array}{l}\text { Superscript *: dimensional quantities; } \\
\text { Subscript } 0 \text { : quantities at initial state; } \\
\mu \text { : dynamic viscosity; } \\
\rho: \text { liquid density; } \\
\kappa: \text { liquid thermal diffusivity; } \\
h \text { : surface heat flux temperature gradient; } \\
\beta \text { : temperature gradient; } \\
\text { d: layer thickness; } \\
\xi: \text { volumetric thermal expansion; } \\
\text { g: gravitational acceleration; } \\
\gamma: \text { temperature variation of surface tension; } \\
\gamma^{\prime}: \text { air-liquid interfacial tension; } \\
\text { V: characteristic velocity; }\end{array}$} \\
\hline Marangoni Number & $M a=\frac{\beta d^{2} \gamma_{0}^{*}}{\kappa_{0}^{*} \mu_{0}^{*}}$ & \\
\hline Capillary Number & $C a=\frac{\mu_{0}^{*} V}{\gamma^{\prime}}$ & \\
\hline
\end{tabular}



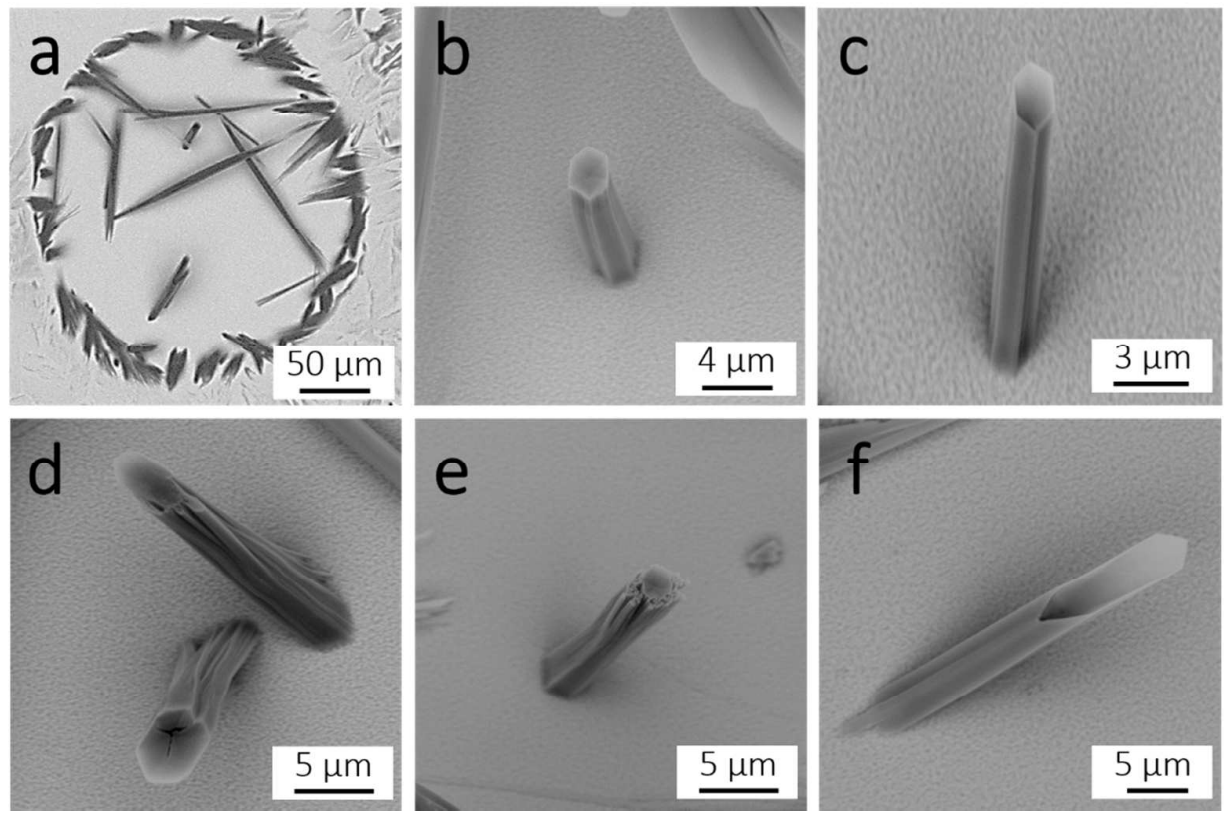

Figure S1. (a) SEM image of an unstable droplet in which discrete peptide rods are formed. (b-f) SEM images of several typical FF nanotubes/rods formed from plumes in a turbulent solution. 

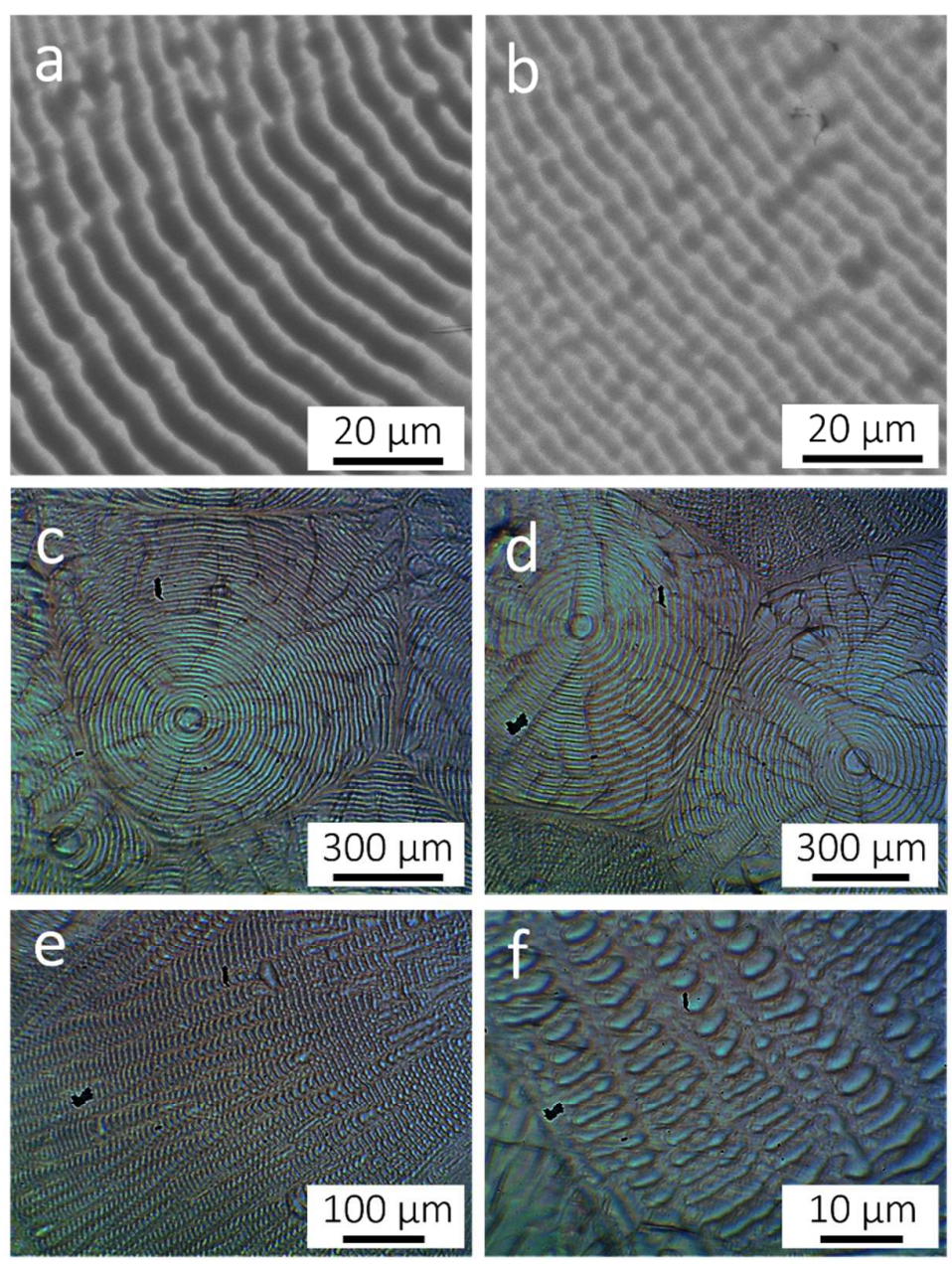

Figure S2. (a) and (b) SEM images of the belts in FF self-assembled concentric patterns at different evaporation rates.

(c) and (d) Optical images of adjoining concentric patterns. (e) and (f) Optical images showing details of the patterns consisting of both radial and concentric bumps. 

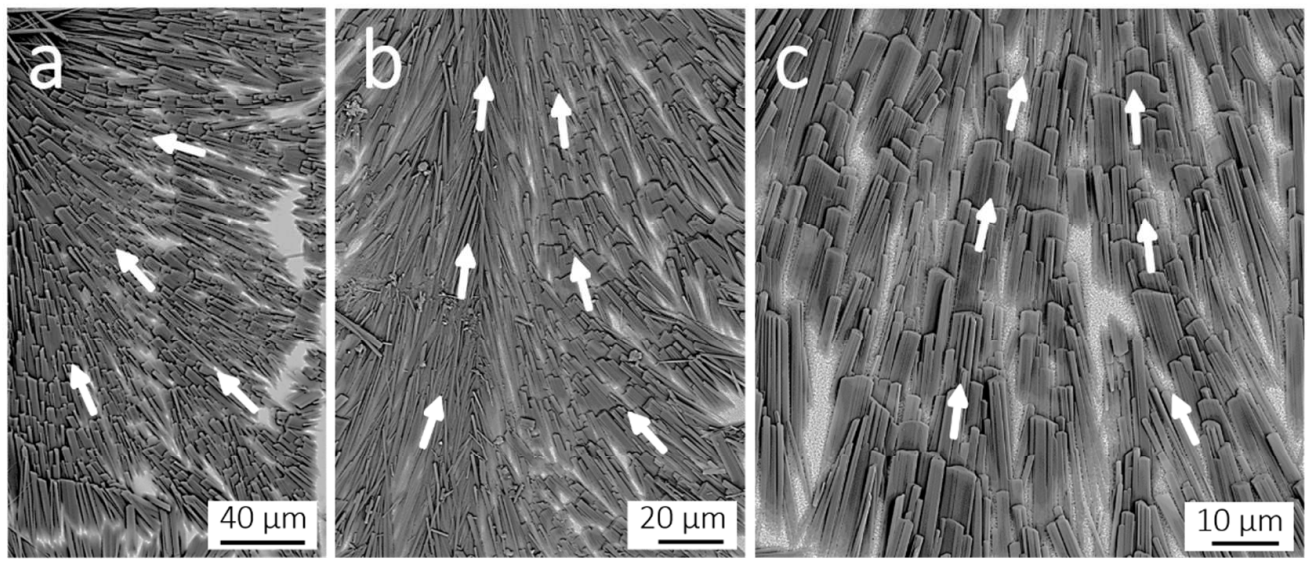

Figure S3. (a-c) SEM images illustrating inclined flake self-assembly along the paths in which the FF/HFIP liquid front moves. The white arrows indicate the direction of the movement of the liquid front. 


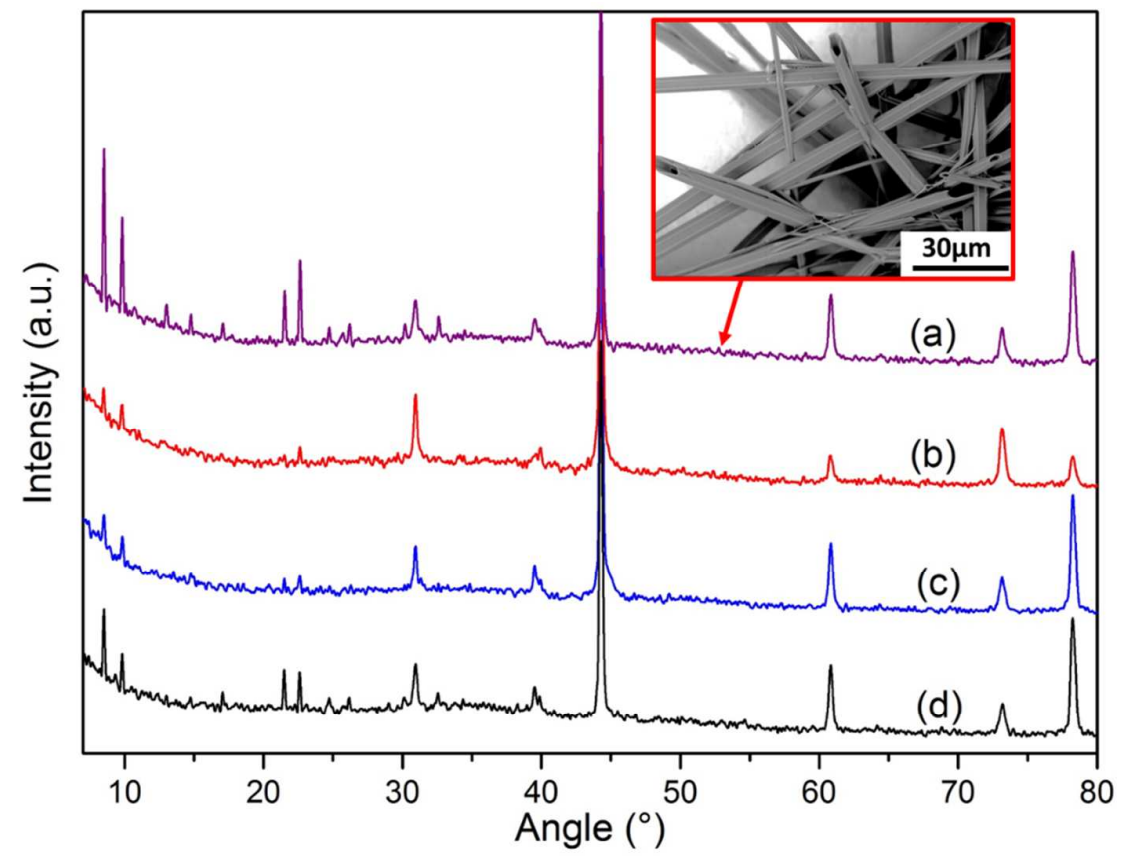

Figure S4. XRD patterns: (a) Interconnected hexagonal FF microtubes, (b) Converged fibers in Figure 3d, (c) Concentric pattern in Figure 4e, and (d) Inclined flakes in Figure 5f. Inset: SEM image of the interconnected hexagonal microtubes. 


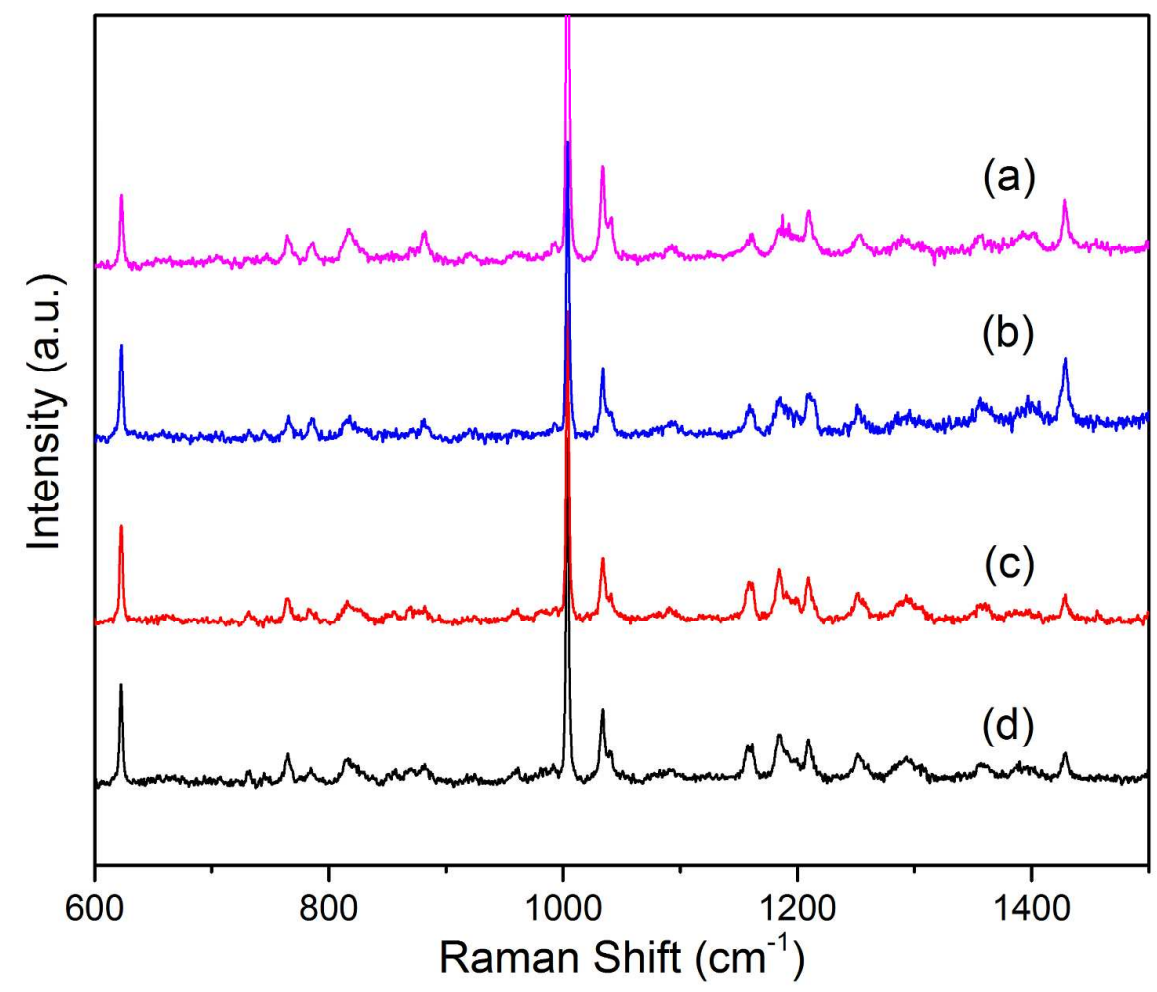

Figure S5. Raman scattering spectra: (a) Interconnected hexagonal FF microtubes, (b) Converged fibers in Figure 3d, (c) Concentric pattern in Figure 4e, and (d) Inclined flakes in Figure 5f. 